\title{
Relativistic path integral and relativistic Hamiltonians in QCD and QED
}

\author{
Yu.A.Simonov, \\ Institute of Theoretical and Experimental Physics \\ 117118, Moscow, B.Cheremushkinskaya 25, Russia
}

\begin{abstract}
The proper-time $4 \mathrm{~d}$ path integral is used as a starting point to derive the new explicit parametric form of the quark-antiquark Green's function in gluonic and QED fields, entering as a common Wilson loop. The subsequent vacuum averaging of the latter allows to derive the instantaneous Hamiltonian. The explicit form and solutions are given in the case of the $q \bar{q}$ mesons in magnetic field.
\end{abstract}

The path-integral formalism in quantum mechanics, created by Feynman [1, 2] is an important benchmark in the development and in our understanding of quantum theory. Many varieties of this formalism and new methods to solve the problems, which seemed before unsolvable, have been suggested since then, see the books and review papers $[3,4,5,6,7,8,9]$, summarizing the modern achievements in this field.

The extension of the path-integral formalism to the quantum field theory was done in several directions. One of the most known line of development was started already in [2], where field variables, i.e. electromagnetic potentials $A_{i}(\mathbf{x}, t), \varphi(\mathbf{x}, t)$ play the role of quantum spacial coordinates $q(t)$, and, the resulting path integral is becoming the functional integral. This line is now a part of standard lore, present in many textbooks, see e.g. [5, 6, 7, 8, , 9].

It is however important, that in all works of this direction the path integration concerns spacial coordinates and/or field variables, but not the time 
coordinate, and in this way one can say, that this development is similar to the path integrals in quantum mechanics, where time plays the ordering role and stays outside of the realm of fluctuating variables.

Another and more general approach, unifying space and time coordinates in the path integral, is based on the proper time coordinate. The latter was introduced by V.Fock 10, and J.Schwinger [11, who used proper time formalism for the field theory in external electromagnetic fields, however did not exploit path integrals.

In QED path integrals, based on the proper time, were suggested in [12] and developed in [13].

Path integrals for QCD both in time and space variables, using proper time as an ordering variable were suggested in [14, 15, 16]. The first use of the QCD path integral for quarks and gluons was done in [17] and exploited to demonstrate the confinement due to field correlators (stochastic confinement) for a review see [18].

The full form of the path integral in QCD for quarks and gluons, based on the proper time ordering, was given in [17] for $T=0$ and in [19] for $T>0$, and different approximations were reviewed in [20] for some relativistic models and in 21] for QCD. It was called the Fock-Feynman-Schwinger representation (FFSR) and we retain this name in what follows.

Based on FFSR a new relativistic Hamiltonian was derived in [22, 23. for quarks and in [24, 25] for gluons, where a new important variable was introduced $-\omega$, playing the role of the einbein variable [26]. Its average value $\omega_{0}$ is the average quark (or gluon) energy and explains the appearance of the notion of constituent mass in earlier models. The relativistic Hamiltonian with einbeins $\omega_{i}$ allows to calculate all low-lying states in QCD: mesons, glueballs, baryons and hybrids from the first-principle input: current quark masses, $\alpha_{s}$ and string tension $\sigma$, see [27, 28, 29] for reviews.

However, the introduction of $\omega_{i}$ as einbein variables, being successful, is an approximate procedure, and its limitations and corrections were not enough clarified in the literature. An attempt in this direction was done in [30], where the fluctuation of the time coordinate in the path integration was substituted by the fluctuations integration in $\Delta \omega_{i}$. The resulting expressions for quark decay constants of mesons in [30] are quite successful in comparison with experiment, however the exact scheme of approximations was not clearly stated.

An additional impulse for a development in this area was given recently by the inclusion of high magnetic field $B$ in the dynamics of QCD and QED, 
see [31, 32, 33, 34, 35] for a recent papers. In this case $\omega_{i}$ depend on $B$ and might vanish or grow fast (depending on quark spin projection), which calls for a careful analysis of all corrections.

In the present paper we undertake such an analysis and rederive different forms of path integrals and relativistic Hamiltonians for the QCD and QED systems, typically for quark-antiquark or atoms, taking into account both QCD and QED dynamics in the first case.

The first thing we meet confronting $4 d$ path integrals, is the problem of the time-coordinate fluctuation, which necessarily requires distinguishing average (ordering) time and fluctuating time, similar to the old notion of the Zitterbewegung. We analyze this phenomenon, comparing the Bethe-Salpeter and path-integral formalisms and show how the latter can be developed using the fact, that all dynamics is contained in the Wilson loop formalism augmented by spin insertions.

\section{Path integral: treating time fluctuations}

We start with the simplest example of a scalar particle in external field, this problem was considered for QED by Feynman in [12].

The scalar one particle Green's function is (in Euclidean space-time)

$$
g(x, y)=\left(\frac{1}{m^{2}-D_{\mu}^{2}}\right)_{x y}=\int_{0}^{\infty} d s\left(D^{4} z\right)_{x y} \exp (-K) \Phi(x, y)
$$

where $D_{\mu}=\partial_{\mu}-i e A_{\mu}$,

$$
\begin{aligned}
& K=m^{2} s+\frac{1}{4} \int_{0}^{s} d \tau\left(\frac{d z_{\mu}}{d \tau}\right)^{2} \\
& \Phi(x, y)=\exp i e \int_{y}^{x} A_{\mu} d z_{\mu},
\end{aligned}
$$

and

$$
\left(D^{4} z\right)_{x y} \simeq \lim _{N \rightarrow \infty} \prod_{n=1}^{N} \int \frac{d^{4} z(n)}{(4 \pi \varepsilon)^{2}} \int \frac{d^{4} p}{(2 \pi)^{4}} e^{i p\left(\sum_{n=1}^{N} z(n)-(x-y)\right)}, N \varepsilon=s .
$$

At this point it is important to stress the difference between the nonrelativistic quantum-mechanical and relativistic path integration: in the first 
case one has $\left(D^{3} z\right)=\left(D^{3} z(t)\right)$ in (11) and the time variable $t$ has the ordering character: the consecutive pieces of trajectory $\mathbf{z}(t)$ are ordered by time. In the relativistic path integral this role is given to the proper time $\tau, s$ while the "time" $z_{4}(\tau)$ is fluctuating together with spacial coordinates $\mathbf{z}(\tau)$. In terms of any local field theory and Bethe-Salpeter type of equation this is allowable and necessary, since any moment of time $z_{4}$ appears in the amplitude with a new interaction point, which may happen before or after the previous interaction point, thus the points of interaction lie chaotically on the time axis. However, from the point of view of a stationary process, which creates the system with a given quantized energy state in the limit of long time interval, one may think of an averaged progressive time and averaged trajectories of constituents, where stochastic time fluctuations are dealt with in a well defined averaging process. In this way the time-fluctuating relativistic trajectories are averaged into stationary time-ordered trajectories, similar to the quantum mechanical ones, where fluctuations are allowed for spacial coordinates. Correspondingly one can write

$$
z_{4}(\tau)=\bar{z}_{4}(\tau)+\tilde{z}_{4}(\tau)
$$

where $\bar{z}_{4}(\tau) \equiv t_{E}=2 \omega \tau$ is the averaged time, proportional to the proper time, while the fluctuating time $\tilde{z}_{4}(\tau)$ can be written as a sum of one-step fluctuations:

$$
\tilde{z}_{4}(\tau)=\sum_{k=1}^{n} \Delta z_{4}(k), \quad \tau=n \varepsilon, \quad N \varepsilon=s, \quad \sum_{k=1}^{N} \Delta z_{4}(k)=0 .
$$

The proper time $s$ is expressed via the total Euclidean time $T=x_{4}-y_{4}$ and the new variable $\omega$,

$$
s=T / 2 \omega,
$$

and hence the scalar Green's function (11) can be rewritten in the form

$$
g(x, y)=T \int_{0}^{\infty} \frac{d \omega}{2 \omega^{2}} D^{3} z e^{-K(\omega)}\langle\Phi(x, y)\rangle_{\Delta z_{4}} .
$$

Here

$$
K(\omega)=\int_{0}^{T} d t_{E}\left(\frac{\omega}{2}+\frac{m^{2}}{2 \omega}+\frac{\omega}{2}\left(\frac{d \mathbf{z}}{d t_{E}}\right)^{2}\right)
$$

while

$$
\langle\Phi(x, y)\rangle_{\Delta z_{4}}=D \Delta z_{4} \exp \left[i e \int A_{i}\left(\mathbf{z}\left(t_{E}\right), t_{E}+\tilde{z}_{4}\right) d z_{i}+i e \int A_{4} d t_{E}+i e \int A_{4} d \Delta z_{4}\right]
$$




$$
D \Delta z_{4} \equiv \int \frac{d p_{4}}{2 \pi} \prod_{k=1}^{n} \frac{d \Delta z_{4}(k)}{\sqrt{4 \pi \varepsilon}} \exp \left\{\sum_{k=1}^{N}\left[i p_{4} \Delta z_{4}(k)-\frac{1}{4} \frac{\left(\Delta z_{4}(k)\right)^{2}}{\varepsilon}+i e \Delta z_{4}(k) A_{4}\right]\right\}
$$

The result of integration in (11) can be written as

$$
\langle\Phi(x, y)\rangle_{\Delta z_{4}}=\sqrt{\frac{\omega}{2 \pi T}} \overline{\Phi(x, y)}
$$

where $\bar{\Phi}(x, y)$ is the averaged Wilson line, augmented by the fluctuation,

$$
\bar{\Phi}(x, y)=\exp \left[i e \int_{\mathbf{y}}^{\mathbf{x}} A_{i}\left(\mathbf{z}\left(t_{E}\right), t_{E}\right) d \mathbf{z}_{i}+i e \int_{y_{4}}^{x_{4}} A_{4}\left(\mathbf{z}\left(t_{E}\right), t_{E}\right) d t_{E}\right] \exp (\Delta S) .
$$

In the simplest case of the free scalar Green's function $A_{\mu} \equiv 0$ and $\bar{\Phi}(x, y)=1$, hence

$$
\begin{gathered}
g_{0}(x, y)=\sqrt{\frac{T}{8 \pi}} \int_{0}^{\infty} \frac{d \omega}{\omega \sqrt{\omega}}\left(D^{3} z\right) \mathbf{x y} e^{-K(\omega)}= \\
=\frac{1}{8 \pi^{2} T} \int_{0}^{\infty} d \omega \exp \left[-\frac{m^{2} T}{2 \omega}-\frac{(\mathbf{x}-\mathbf{y})^{2}}{2 T} \omega-\frac{\omega T}{2}\right]=\frac{1}{4 \pi^{2}} \frac{m}{u} K_{1}(m u), u^{2}=T^{2}+(\mathbf{x}-\mathbf{y})^{2},
\end{gathered}
$$

where $K_{1}(x)$ is the Bessel function of the second kind.

At this point the role of $\omega$ becomes clear, since for large $T$ the integral in (14) can be taken by the stationary point method with the action

$$
S(\omega, T)=\frac{m^{2} T}{2 \omega}+\frac{(\mathbf{x}-\mathbf{y})^{2}}{2 T} \omega+\frac{\omega T}{2} ;\left.\quad \frac{\partial S(\omega, T)}{\partial \omega}\right|_{\omega=\omega_{0}}=0,
$$

and

$$
\omega_{0}=\frac{m T}{\sqrt{\left(\mathbf{x}^{2}-\mathbf{y}\right)^{2}+T^{2}}} \rightarrow m, \text { for } T \gg|\mathbf{x}-\mathbf{y}|,
$$

and one finally obtains the standard answer for large $T$ yielding the asymptotics of the r.h.s. of (14) at large $T$.

$$
g_{0}(x, y)=\frac{\sqrt{m}}{4 \pi^{3 / 2} T^{3 / 2}} \exp (-m T)
$$




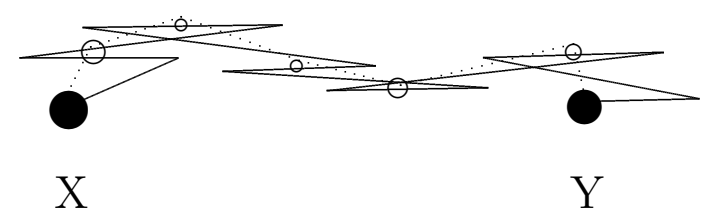

Figure 1: The time-fluctuating trajectory in the $\left(z_{1}, z_{4}\right)$ plane. The points $\left(z_{1}\left(t_{E}\right), t_{E}\right)$ are marked by circles and are connected by average trajectory, depicted by dotted line.

Another form exploits the Hamiltonian in (14), namely, one can use the relation

$$
\int\left(D^{3} z\right) \mathbf{x y} e^{-K(\omega)}=\left\langle\mathbf{x}\left|e^{-H(\omega) T}\right| \mathbf{y}\right\rangle
$$

where

$$
H(\omega)=\frac{\mathbf{p}^{2}+m^{2}}{2 \omega}+\frac{\omega}{2}
$$

Applying the stationary point method to the integrals (14), (17), one obtains at large $T$ the energy eigenvalue

$$
\frac{\partial H(\omega)}{\partial \omega} \mid \omega=\omega_{0}=0 ; \quad \omega_{0}=\sqrt{\mathbf{p}^{2}+m^{2}} .
$$

From (19) one can understand, that $\omega$ plays the role of a virtual particle energy, and the condition (19) has the meaning of the energy shell condition. This interpretation holds also for the case of $N$ particles with interaction, when the integrals aver $\prod_{i=1}^{N} d \omega_{i}$ are involved. Note, that in this way $\omega_{i}$ are not any more approximate einbein variables, as in our previous works see e.g. [18, 23].

We now turn to the case of $A_{\mu} \neq 0$ and remark, that $A_{\mu}\left(\mathbf{z}, z_{4}\right)$ are functions of coordinates, which will be used later in the process of vacuum averaging, yielding points of interaction, correlators etc., but at this moment in (13), $\bar{\Phi}(x, y)$ is a set of all possible Wilson lines, obtained by time fluctuations with the weight, given in (11), see Fig.1 as an illustration.

In one particular case, when $\mathbf{z}\left(t_{E}\right)$ is fixed, i.e. the trajectory is parallel to the $z_{4}$ axis, all $\Delta z_{4}$ fluctuations are washed out, since all fluctuations cancel each other,

$$
\exp \left(i e \int A_{4}\left(\mathbf{z}, z_{4}\right) d z_{4}\right)=\exp \left(i e \int A_{4}\left(\mathbf{z}, t_{E}\right) d t_{E}\right)
$$


The same would happen in the case of QCD, where again overlapping pieces of Wilson line cancel each other. We shall come back to the problem of fluctuating Wilson lines, when we consider gauge invariant two-body Green's functions.

In the case of the white system of the quark and antiquark of opposite charges, one must start with the one-body Green's function

$S_{i}(x, y)=\left(m_{i}+\hat{\partial}-i g \hat{A}-i e_{i} \hat{A}^{(e)}\right)_{x y}^{-1} \equiv\left(m_{i}+\hat{D}^{(i)}\right)_{x y}^{-1}=\left(m_{1}-\hat{D}^{(i)}\right)\left(m_{i}^{2}-\left(\hat{D}^{(i)}\right)^{2}\right)_{x y}^{-1}$

The path-integral representation for $S_{i}$ is [8]

$$
S_{i}(x, y)=\left(m_{i}-\hat{D}^{(i)}\right) \int_{0}^{\infty} d s_{i}(D z)_{x y} e^{-K_{i}} \Phi_{\sigma}^{(i)}(x, y) \equiv\left(m_{i}-\hat{D}^{(i)}\right) G_{i}(x, y),
$$

where

$$
\begin{gathered}
K_{i}=m_{i}^{2} s_{i}+\frac{1}{4} \int_{0}^{s_{i}} d \tau_{i}\left(\frac{d z_{\mu}^{(i)}}{d \tau_{i}}\right)^{2} \\
\Phi_{\sigma}^{(i)}(x, y)=P_{A} P_{F} \exp \left(i g \int_{y}^{x} A_{\mu} d z_{\mu}^{(i)}+i e_{i} \int_{y}^{x} A_{\mu}^{(e)} d z_{\mu}^{(i)}\right) \times \\
\times \exp \left(\int_{0}^{s_{i}} d \tau_{i} \sigma_{\mu \nu}\left(g F_{\mu \nu}+e_{i} B_{\mu \nu}\right)\right) .
\end{gathered}
$$

Here $F_{\mu \nu}$ and $B_{\mu \nu}$ are correspondingly gluon and c.m. field tensors, $P_{A}, P_{F}$ are ordering operators, $\sigma_{\mu \nu}=\frac{1}{4 i}\left(\gamma_{\mu} \gamma_{\nu}-\gamma_{\nu} \gamma_{\mu}\right)$. Eqs. (21-24) hold for the quark, $i=1$, while for the antiquark one should reverse the signs of $e_{i}$ and g. In explicit form one writes

$$
\sigma_{\mu \nu} F_{\mu \nu}=\left(\begin{array}{ll}
\boldsymbol{\sigma} \mathbf{H} & \boldsymbol{\sigma} \mathbf{E} \\
\boldsymbol{\sigma} \mathbf{E} & \boldsymbol{\sigma} \mathbf{H}
\end{array}\right), \quad \sigma_{\mu \nu} B_{\mu \nu}=\left(\begin{array}{ll}
\boldsymbol{\sigma} \mathbf{B} & 0 \\
0 & \boldsymbol{\sigma} \mathbf{B}
\end{array}\right) .
$$

The two-body $q_{1} q_{2}$ Green's function can be written as [17, 21]

$$
\begin{gathered}
G_{q_{1} \bar{q}_{2}}(x, y)=\int_{0}^{\infty} d s_{1} \int_{0}^{\infty} d s_{2}\left(D z^{(1)}\right)_{x y}\left(D z^{(2)}\right)_{x y}\left\langle\hat{T} W_{\sigma}(A)\right\rangle_{A} \times \\
\times \exp \left(i e_{1} \int_{y}^{x} A_{\mu}^{(e)} d z_{\mu}^{(1)}-i e_{2} \int_{y}^{x} A_{\mu}^{(e)} d z_{\mu}^{(2)}+e_{1} \int_{0}^{s_{1}} d \tau_{1}(\boldsymbol{\sigma} \mathbf{B})-e_{2} \int_{0}^{s_{2}} d \tau_{2}(\boldsymbol{\sigma} \mathbf{B})\right),
\end{gathered}
$$

where

$$
\hat{T}=\operatorname{tr}\left(\Gamma_{1}\left(m_{1}-\hat{D}_{1}\right) \Gamma_{2}\left(m_{2}-\hat{D}_{2}\right)\right)
$$


"tr" is the trace over Dirac and color indices acting on all terms. Here $\left\langle W_{\sigma}(A)\right\rangle$ is the closed Wilson loop with the spin insertions and one should have in mind, that color and e.m. spin insertions in general do not commute, which should be taken into account when computing spin-dependent part of interaction, see [36], in (26) this fact was disregarded.

$$
W_{\sigma}(A)=P_{a} P_{F} \exp \left[i g \oint A_{\mu} d z_{\mu}+g \int_{0}^{s_{1}} \sigma_{\mu \nu}^{(1)} F_{\mu \nu} d \tau_{1}-g \int_{0}^{s_{2}} \sigma_{\mu \nu}^{(2)} F_{\mu \nu} d \tau_{2}\right] \text {. }
$$

It is important, that the physically meaningful result for the Green's function is obtained by two different averaging procedures applied to the total Wilson loop $W=\Phi_{\sigma}^{(1)}(x, y) \Phi_{\sigma}^{(2)}(y, x)$ :

1) one should overage $W$ over all time fluctuations ;

2) one should average $W$ over all nonperturbative (np) and perturbative (pert) field configurations with the weight, given by the standard QED+QCD field actions, so that the final result is

$$
\langle\langle W\rangle\rangle \equiv\left\langle\langle W\rangle_{\Delta z_{4}}\right\rangle_{A, A^{(e)}}
$$

However, the class of processes of interest in QCD is very wide, since any process, starting and finishing with definite hadron states, such as formfactors, decays, hadron reactions, needs an explicit definition of initial and final states as eigenstates of the Hamiltonian $H\left(\omega_{1}, \omega_{2}\right)$, and therefore can use the formalism, discussed in this paper.

It is clear, that in the fluctuation averaging $\langle W\rangle_{\Delta z_{4}}$ the result is an average Wilson loop, passing through the points $\left\{\mathbf{z}\left(t_{E}\right)+\Delta \mathbf{z}\left(t_{E}\right), t_{E}+\Delta t_{E}\right\}, t_{E} \epsilon(0, T)$, where $\Delta \mathbf{z}\left(t_{E}\right), \Delta t_{E}$ depend on $T, m_{1}, m_{2}$ and also on the concrete field configuration, which will be averaged in the next averaging process, over vacuum fields.

One can estimate the average time fluctuation $\Delta t_{E}$ in the case of the free relativistic particle propagation.

E.g. assuming the correlation function to have the form

$$
f\left(z_{4}^{(1)}-z_{4}^{(2)}\right)=\exp \left(-\frac{\left(z_{4}^{(1)}-z_{4}^{(2)}\right)^{2}}{(\Delta \bar{z})^{2}}\right),
$$

and taking into account time fluctuations $z_{4}^{(1)}=t_{E}^{(1)}+\tilde{z}_{4}\left(t_{E}\right)$, and integrating over $\Delta z_{4}$, one obtains the increase of the correlation time

$$
(\Delta \bar{z})^{2} \rightarrow(\Delta z)_{\Delta z_{4}}^{2}=(\Delta \bar{z})^{2}+\frac{t_{E}^{(1)}}{2 \omega} \sim(\Delta \bar{z})^{2}+\frac{T}{2 m} .
$$


However this result is an artefact of the not accurate definition of the path-integration measure, when at the ends of the time interval $\Delta t_{E}$ the path can change the direction, implying infinite time derivative. Imposing a proper condition on the magnitude of the derivative, i.e. with smooth trajectories, the result would be different. From the point of view of the relation $\Delta M \Delta t \gtrsim 1$, one can in principle calculate however accurate values of masses $M$ for large $T$, and only the coupling to decay channels, i.e. the width $\Gamma$ should put a lower limit on the accuracy $\Delta M$.

It is interesting, how this problem occurs in our path-integral formalism. Indeed, the basic dynamics which is contained in $\langle\langle W\rangle\rangle$, when time fluctuation is supported by interaction, Eq. (30), can be described by the diagram in Fig.2. Now, from the point of view of Hamiltonian dynamics with the trace of the hypersurface, shown in Fig.2 by a dotted line, the Hamiltonian becomes a matrix, with Fock states, numerating columns and rows,

$$
H_{q \bar{q}} \rightarrow\left(\begin{array}{lll}
H_{q \bar{q}} & \hat{V}_{12} & \ldots \\
\hat{V}_{21} & H_{(q \bar{q}),(q \bar{q})_{2}} & \ldots \\
\ldots & \ldots & \ldots
\end{array}\right),
$$

where nondiagonal elements are transition operators and diagonal ones define dynamics (and masses) of more and more complex systems. Therefore, e.g. $\hat{V}_{13}$ is responsible for the decay $q \bar{q} \rightarrow(q \bar{q})_{1}+(q \bar{q})_{2}$, and hence defines the accuracy of possible mass determination of the incident state $(q \bar{q})$.

The wave functions of (32) are actually Fock columns of different states,

e.g. $\left\{\Psi_{q \bar{q}}, \Psi_{(q \bar{q})(q \bar{q}), \ldots}\right\}$, and therefore the $(q \bar{q})$ eigenstates $\left\{\Phi_{q \bar{q}}^{(n)}\right\}$ are not any more an orthonormal set of states. As we shall see, the eigenstates $\Psi_{n}\left(\omega_{1}, \omega_{2}\right)$ will not be orthonormal on the energy shells $\left(\omega_{1}^{(0)}, \omega_{2}^{(0)}\right)$, different for each $n$. In this way going from $4 \mathrm{~d}$ path integral to the relativistic Hamiltonian $3 \mathrm{~d}$ formalism one naturally meets the many-channel Hamiltonian, where diagonal elements correspond to the fluctuation-averaged trajectories.

\section{From path integral to instantaneous dy- namics}

As a result of two averaging processes; time fluctuation and vacuum averaging the basic dynamical input of the resulting $3 \mathrm{~d}$ path integral - the doubly 


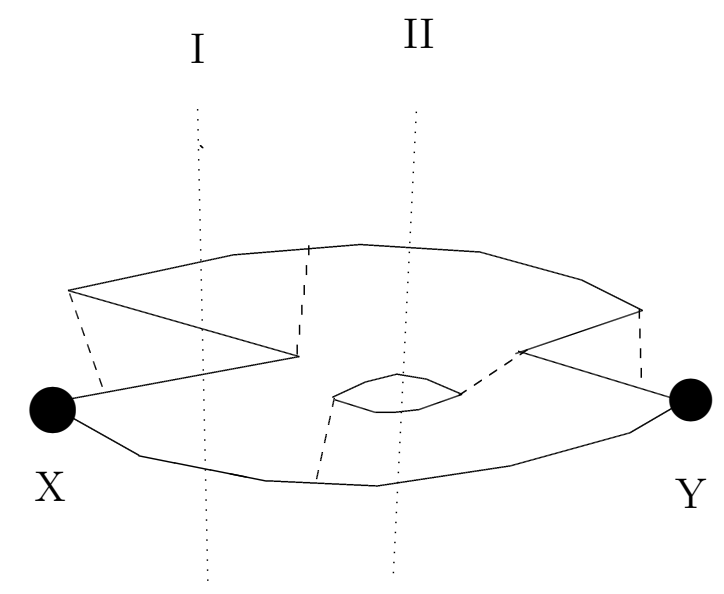

Figure 2: The vacuum averaged Wilson lines, displaying pair creation in the time fluctuation process. The hypersurface traces I and II mark the double quark pair state of the Hamiltonian

renormalized Wilson loop can be written as

$$
\begin{gathered}
\langle\langle W\rangle\rangle=Z_{W} \exp \left\{-\frac{1}{2} \iint d \pi_{\mu \nu}(1) d \pi_{\lambda \sigma}(2)\left[g^{2}\left\langle F_{\mu \nu}(1) F_{\lambda \sigma}(2)\right\rangle+\right.\right. \\
\left.\left.e^{2}\left\langle F_{\mu \nu}^{(e)}(1) F_{\lambda \sigma}^{(e)}(2)\right\rangle\right]+O(F F F)\right\}
\end{gathered}
$$

where

$$
d \pi_{\mu \nu} \equiv d s_{\mu \nu}+\sigma_{\mu \nu}^{(1)} \frac{d t_{E}^{(1)}}{2 \omega_{1}}-\sigma_{\mu \nu}^{(2)} \frac{d t_{E}^{(2)}}{2 \omega_{2}},
$$

and the integration $d s_{\mu \nu}$ is done over the minimal area $S_{\text {min }}$ inside the timeaveraged trajectories of quark and antiquark $\bar{L}_{1}$ and $\bar{L}_{2}$. Note, that in addition to the time-fluctuation smearing discussed above, there is also nonperturbative smearing, provided by the np field correlators.

Indeed, the quadratic (Gaussian) color field correlators can be written as [19]

$$
\frac{g^{2}}{N_{c}}\left\langle\left\langle\operatorname{Tr} E_{i}(x) \Phi E_{j}(y) \Phi^{\dagger}\right\rangle\right\rangle=\delta_{i j}\left(D^{E}(u)+D_{1}^{E}(u)+u_{4}^{2} \frac{\partial D_{1}^{E}}{\partial u^{2}}\right)+u_{i} u_{j} \frac{\partial D_{1}^{E}}{\partial u^{2}}
$$




$$
\begin{gathered}
\frac{g^{2}}{N_{c}}\left\langle\left\langle\operatorname{Tr} H_{i}(x) \Phi H_{j}(y) \Phi^{\dagger}\right\rangle\right\rangle=\delta_{i j}\left(D^{H}(u)+D_{1}^{H}(u)+\mathbf{u}^{2} \frac{\partial D_{1}^{H}}{\partial \mathbf{u}^{2}}\right)-u_{i} u_{j} \frac{\partial D_{1}^{H}}{\partial u^{2}} \\
\frac{g^{2}}{N_{c}}\left\langle\left\langle\operatorname{Tr} H_{i}(x) \Phi E_{j}(y) \Phi^{\dagger}\right\rangle\right\rangle=\varepsilon_{i j k} u_{4} u_{k} \frac{\partial D_{1}^{E H}}{\partial u^{2}}
\end{gathered}
$$

where $D^{E}, D^{H}$ are purely np correlators, and $D_{1}^{E, H}$ contain perturbative part. The same type of equations, but with replacement $\frac{g^{2}}{N_{c}} \rightarrow e^{2}$ and keeping only $D_{1}^{E, H}$ holds also for e.m. correlators. Note, that at zero temperature colorelectric and colormagnetic correlators coincide, note also that np correlators $D^{E}, D^{H}$ are due to Euclidean vacuum fields.

The explicit form of perturbative correlators $D_{1}^{E, H}$ to lowest order in $\alpha_{s}$ is

$$
D_{1}^{E}(x)=D_{1}^{H}(x)=\frac{16 \alpha_{s}}{3 \pi x^{4}}+O\left(\alpha_{s}^{2}\right),
$$

while for e.m. fields one should replace $\frac{4}{3} \alpha_{s} \rightarrow \alpha$.

At this point it is important to realize, that the correlators depend on space and time intervals, e.g. $D\left(\mathbf{z}^{(1)}-\mathbf{z}^{(2)}, t_{E}^{(1)}-t_{E}^{(2)}\right)$ and $\langle\langle W\rangle\rangle$ in Eq. (34) even after fluctuation averaging implies nonlocal in time dynamics, e.g. the term $\iint e^{z}\left\langle F_{\mu \nu}^{(e)}(1) F_{\lambda \sigma}^{(e)}(2)\right\rangle d s_{\mu \nu}(1) d s_{\lambda \sigma}(2)$ stands actually for a photon exchange diagram. We are now going to replace this time nonlocal interaction by the instantaneous one, which is easily done in the correlator language, simply by integrating in (34) all correlators over time differences, $t_{E}^{(1)}-t_{E}^{(2)}$,

$$
d t_{E}^{(1)} d t_{E}^{(2)}=d t_{E} d\left(t_{E}^{(1)}-t_{E}^{(2)}\right), d t_{E}=d \frac{t_{E}^{(1)}+t_{E}^{(2)}}{2} .
$$

It is important, that the main part of our interaction, the confining interaction, is ensured by the correlator $D^{E}\left(\sqrt{t^{2}+\mathbf{r}^{2}}\right)$, which has a very small correlation length $\lambda$, as was shown on the lattice [36] and analytically [37]. $D^{E}(t) \sim e^{-t / \lambda}, t \gtrsim \lambda, \quad \lambda \sim 0.1 \mathrm{fm}$, and therefore the transition to the instantaneous dynamics is done on small averaging interval $\Delta t \sim \lambda$. Therefore for all processes with momentum (energy) transfer $\Delta Q$, satisfying $\Delta Q \lambda \lesssim 1$, this transition of np confining mechanism to the instantaneous dynamics is allowable. The case of gluon exchange is similar to the Coulomb interaction, where the instantaneous approximation in the Bethe-Salpeter equation is known as the Salpeter equation and is widely used in the literature. We shall mostly use the one-gluon exchange (OGE) interaction as a perturbation and therefore our transition to the instantaneous dynamics is justified. 
For the case of the zero angular momentum (see in [23] the general derivation) one can write for the instantaneous straight line $w_{\mu}(t, \beta)=z_{\mu}^{(1)}(t) \beta=$ $z_{\mu}^{(2)}(t)(1-\beta), 0 \leq \beta \leq 1$, and e.g. $d s_{\mu 4}=\left(z_{\mu}^{(1)}(t)-z_{\mu}^{(2)}(t)\right) d \beta d t$.

For zero angular momentum one can simplify the integration over the area of the minimal surface in (33) and obtain the result, neglecting spincontaining terms in (33) for the moment,

$$
\left.\langle\langle W\rangle\rangle=Z_{W} \exp \left(-\int_{0}^{T}\left[V_{0}\left(r\left(t_{E}\right)\right)\right]\right) d t_{E}\right),
$$

where $r\left(t_{E}\right)=\left|\mathbf{z}_{1}\left(t_{E}\right)-\mathbf{z}_{2}\left(t_{E}\right)\right|$, and

$$
\begin{gathered}
V_{0}(r)=V_{\text {conf }}(r)+V_{O G E}(r), \\
V_{\text {conf }}(r)=2 r \int_{0}^{r} d \lambda \int_{0}^{\infty} d \nu D(\lambda, \nu) \rightarrow \sigma r,(r \rightarrow \infty), \\
\sigma=2 \int_{0}^{\infty} d \nu \int_{0}^{\infty} d \lambda D(\nu, \lambda), \\
V_{O G E}=\int_{0}^{r} \lambda d \lambda \int_{0}^{\infty} d \nu D_{1}^{\text {pert }}(\lambda, \nu)=-\frac{4}{3} \frac{\alpha_{s}}{r}
\end{gathered}
$$

As a result one can write for the product of $q \bar{q}$ Green's functions (we omit renormalization $Z$ factors, Fock amplitude coefficients, and ordering operators for simplicity)

$$
\left(\frac{1}{\left(m_{1}^{2}-\hat{D}_{1}^{2}\right)\left(m_{2}^{2}-\hat{D}_{2}^{2}\right)}\right)_{x y}=\frac{T}{8 \pi} \int_{0}^{\infty} \frac{d \omega_{1}}{\omega_{1}^{3 / 2}} \int_{0}^{\infty} \frac{d \omega_{2}}{\omega_{2}^{3 / 2}}\left(D^{3} z_{1}\right) \mathbf{x y}\left(D^{3} z_{2}\right) \mathbf{x y} e^{-A\left(\omega_{1}, \omega_{2}, \mathbf{z}_{1}, \mathbf{z}_{2}\right)}
$$

where $A \equiv K_{1}\left(\omega_{1}\right)+K_{2}\left(\omega_{2}\right)+\int V_{0}\left(r\left(t_{E}\right)\right) d t_{E}$, and

$$
K_{i}\left(\omega_{i}\right)=\frac{m_{i}^{2}+\omega_{i}^{2}}{2 \omega_{i}} T+\int_{0}^{T} d t_{E} \frac{\omega_{i}}{2}\left(\frac{d \mathbf{z}^{(i)}}{d t_{E}}\right)^{2}
$$

We can also introduce here the two-body $3 \mathrm{~d}$ Hamiltonian $H\left(\omega_{1}, \omega_{2}, \mathbf{p}_{1}, \mathbf{p}_{2}\right)$ and rewrite (41) as

$$
\left(\frac{1}{\left(m_{1}^{2}-\hat{D}_{1}^{2}\right)\left(m_{2}^{2}-\hat{D}_{2}^{2}\right)}\right)_{x y}=\frac{T}{8 \pi} \int_{0}^{\infty} \frac{d \omega_{1}}{\omega_{1}^{3 / 2}} \int_{0}^{\infty} \frac{d \omega_{2}}{\omega_{2}^{3 / 2}}\left\langle\mathbf{x}\left|e^{-H\left(\omega_{1}, \omega_{2}, \mathbf{p}_{1}, \mathbf{p}_{2}\right) T}\right| \mathbf{y}\right\rangle .
$$


where $H$ is obtained in a standard way from the action $A\left(\omega_{1}, \omega_{2}, \mathbf{z}_{1}, \mathbf{z}_{2}\right)$ (we omit all e.m. fields except for external magnetic fields $\mathbf{B}$ )

$$
H=\sum_{i=1}^{2} \frac{\left(\mathbf{p}^{(i)}-\frac{e_{i}}{2}\left(\mathbf{B} \times \mathbf{z}^{(i)}\right)\right)^{2}+m_{i}^{2}+\omega_{i}^{2}-e_{i} \boldsymbol{\sigma}^{i} \mathbf{B}}{2 \omega_{i}}+V_{0}(r)+V_{s s}+\Delta M_{S E}
$$

and $V_{0}$ is given in (37). The spin-dependent part of $H, V_{s s}$ is obtained perturbatively from $\sigma_{\mu \nu} F_{\mu \nu}$ terms in (28), and is calculated in the presence of m.f. in [36]. It is considered as a perturbative correction and is a relativistic generalization of the standard hyperfine interaction,

$$
V_{s s}(r)=\frac{1}{4 \omega_{1} \omega_{2}} \int\left\langle\sigma_{\mu \nu}^{(i)} F_{\mu \nu}(x) \sigma_{\rho \lambda}^{(2)} F_{\rho \lambda}(y)\right\rangle d\left(x_{4}-y_{4}\right) .
$$

Its explicit form is given in [38]. Finally, the correction $\frac{\left\langle\sigma^{(i)} F(x) \sigma^{(i)} F(y)\right\rangle}{4 \omega_{1} \omega_{2}}$, where $i$ refers to the same quark (antiquark) yields the spin-independent self-energy correction $\Delta M_{S E}$ which was calculated earlier [39] and for zero mass quarks and no m.f. is

$$
\Delta M_{S E}=-\frac{3 \sigma}{2 \pi \omega_{1}}-\frac{3 \sigma}{2 \pi \omega_{2}} .
$$

For the case of nonzero m.f. the resulting $\Delta M_{S E}$ is given in [38]. We can now write the total Green's function of $q_{1} \bar{q}_{2}$ system, denoting by $Y$ the product of projection operators $Y=\Gamma\left(m_{1}-\hat{D}_{1}\right) \Gamma\left(m_{2}-\hat{D}_{2}\right)$,

$$
m_{1}-\hat{D}_{1}=m_{1}-i \hat{p}_{1}=m_{1}+\omega_{1} \gamma_{4}-i \mathbf{p} \gamma, m_{2}-\hat{D}_{2}=m_{2}-\omega_{2} \gamma_{4}-i \mathbf{p} \gamma
$$

where $\mathbf{p}$ is the quark 3 momentum in the c.m. system.

As a result one has

$$
\begin{gathered}
\int d^{3}(\mathbf{x}-\mathbf{y}) G(x, y)=\int d^{3}(\mathbf{x}-\mathbf{y}) \operatorname{tr}\left(\frac{4 Y_{\Gamma}}{\left(m_{1}^{2}-\hat{D}_{1}^{2}\right)\left(m_{2}^{2}-\hat{D}_{2}^{2}\right)}\right)_{x y}= \\
=\frac{T}{2 \pi} \int_{0}^{\infty} \frac{d \omega_{1}}{\omega_{1}^{3 / 2}} \int_{0}^{\infty} \frac{d \omega_{2}}{\omega_{2}^{3 / 2}}\left\langle Y_{\Gamma}\right\rangle\left\langle\mathbf{x}\left|e^{-H\left(\omega_{1}, \omega_{2}, \mathbf{p}_{1}, \mathbf{p}_{2}\right) T}\right| \mathbf{y}\right\rangle
\end{gathered}
$$

We have used in (46) the relations $4\langle Y\rangle=\operatorname{tr}\left\langle\Gamma\left(m_{1}-i \hat{p}_{1}\right) \Gamma\left(m_{2}-i \hat{p}_{2}\right)\right)$, and neglect spin dependent terms in $H$; we have taken into account, that $D_{\mu}$ acting on Wilson line, i.e. $D_{\mu} \exp \left(i g \int^{x} A_{\mu} d z_{\mu}\right) \Lambda$ yields $\exp \left(i g \int^{x} A_{\mu} d z_{\mu}\right) \partial_{\mu} \Lambda$. The c.m. projection of the Green's function yields

$$
\int d^{3}(\mathbf{x}-\mathbf{y})\left\langle\mathbf{x}\left|e^{-H\left(\omega_{1}, \omega_{2}, \mathbf{p}_{1}, \mathbf{p}_{2}\right) T}\right| \mathbf{y}\right\rangle=\sum_{n} \varphi_{n}^{2}(0) e^{-M_{n}\left(\omega_{1}, \omega_{2}\right) T}
$$


see Appendix 1 for explicit separation of relative coordinates, Eq. (A.1 9)(A.1 12). Here $M_{n}\left(\omega_{1}, \omega_{2}\right)$ is the eigenvalue of $H\left(\omega_{1}, \omega_{2}, \mathbf{p}_{1}, \mathbf{p}_{2}\right)$ in the c.m. system, where $\mathbf{P}=\mathbf{p}_{1}+\mathbf{p}_{2}=0 ; \quad \mathbf{p}_{1}=\mathbf{p}=-\mathbf{p}_{2}$.

The integrals over $d \omega_{1}, d \omega_{2}$ for $T \rightarrow \infty$ can be performed by the stationary point method, namely one has

$$
\begin{gathered}
\int G(x, y) d^{3}(\mathbf{x}-\mathbf{y})=\frac{T}{2 \pi} \int_{0}^{\infty} \frac{d \omega_{1}}{\omega_{1}^{3 / 2}} \int_{0}^{\infty} \frac{d \omega_{2}}{\omega_{2}^{3 / 2}} \sum_{n} e^{-M_{n}\left(\omega_{1}, \omega_{2}\right) T} \varphi_{n}^{2}(0)\langle Y\rangle \\
=\sum_{n} \frac{e^{-M_{n}\left(\omega_{1}^{(0)}, \omega_{2}^{(0)}\right) T} \varphi_{n}^{2}(0)\langle Y\rangle}{\omega_{1}^{(0)} \omega_{2}^{(0)} \sqrt{\left(\omega_{1}^{(0)} M_{n}^{\prime \prime}(1)\right)\left(\omega_{2}^{(0)} M_{n}^{\prime \prime}(2)\right)}},
\end{gathered}
$$

where

$$
\left.\frac{\partial M_{n}\left(\omega_{1}, \omega_{2}\right)}{\partial \omega_{i}}\right|_{\omega_{i}=\omega_{i}^{(0)}}=0, \quad M_{n}^{\prime \prime}(i)=\left.\frac{\partial M_{n}\left(\omega_{1}, \omega_{2}\right)}{\partial \omega_{i}^{2}}\right|_{\omega_{i}=\omega_{i}^{(0)}},
$$

and we have neglected the mixed terms $\frac{\partial^{2} M_{n}}{\partial \omega_{1} \partial \omega_{2}}$ for simplicity, however should keep them in concrete calculations: see exact result in Appendix 1. Comparing the results (47), (48) with the definitions of quark decay constants $f_{\Gamma}^{n}$

$$
\begin{aligned}
\int G_{\Gamma}(x) d^{3} \mathbf{x} & =\sum_{n} \int d^{3} \mathbf{x}\left\langle 0\left|j_{\Gamma}\right| n\right\rangle\left\langle n\left|j_{\Gamma}\right| 0\right\rangle e^{i \mathbf{P} \mathbf{x}-M_{n} T} \frac{d^{3} \mathbf{P}}{2 M_{n}(2 \pi)^{3}} \\
& =\sum_{n} \varepsilon_{\Gamma} \otimes \varepsilon_{\Gamma} \frac{\left(M_{n} f_{\Gamma}^{n}\right)^{2}}{2 M_{n}} e^{-M_{n} T}
\end{aligned}
$$

where for $\Gamma=\gamma_{\mu}, \gamma_{\mu} \gamma_{5}$

$$
\sum_{k=1,2,3} \varepsilon_{\mu}^{(k)}(q) \varepsilon_{\nu}^{(k)}(q)=\delta_{\mu \nu}-\frac{q_{\mu} q_{\nu}}{q^{2}}
$$

and $\varepsilon_{\Gamma}=1$ for $\Gamma=1, \gamma_{5}$, one obtains the expression for $f_{\Gamma}^{n}$ (to lowest order in $V_{s s}$ )

$$
\left(f_{\Gamma}^{n}\right)^{2}=\frac{N_{c}\left\langle Y_{\Gamma}\right\rangle\left|\varphi_{n}(0)\right|^{2}}{\omega_{1}^{(0)} \omega_{2}^{(0)} M_{n} \xi_{n}}, \quad \xi_{n} \equiv \sqrt{\left(\omega_{1}^{(0)} M_{n}^{\prime \prime}(1)\right)\left(\omega_{2}^{(0)} M_{n}^{\prime \prime}(2)\right)},
$$

It is interesting, that numerical estimates using (52) and (A.1 20) are close to those, obtained in [30]. 


\section{Relativistic Hamiltonians of a meson in mag- netic field}

The resulting relativistic Hamiltonian in the instantaneous limit ia given in (43) and can be written as

$H-\sum_{i=1}^{2} \frac{\left(\mathbf{p}^{(i)}-\frac{e_{i}}{2}\left(\mathbf{B} \times \mathbf{z}^{(i)}\right)\right)^{2}+m_{i}^{2}+\omega_{i}^{2}-e_{i} \boldsymbol{\sigma}^{(i)} B}{2 \omega_{i}}+U\left(\mathbf{z}^{(1)}-\mathbf{z}^{(2)}, \boldsymbol{\sigma}^{(1)}, \boldsymbol{\sigma}^{(2)}, \omega_{1}, \omega_{2}\right)$

where

$$
U=V_{0}(r)+V_{s s}+\Delta M_{S E}
$$

We shall be interested in the spectrum of the $q_{1} \bar{q}_{2}$ system in the magnetic field $\mathbf{B}$, but before that we shall test the general form of the Hamiltonian $H\left(\omega_{1}, \omega_{2}, \mathbf{p}_{1} \mathbf{p}_{2}\right)$ and its eigenvalues, obtained at the stationary point values $\omega_{1}^{(0)}, \omega_{2}^{(0)}$.

We start with the case of $\mathbf{B}=0$ and $U=-\frac{Z \alpha}{\left|\mathbf{Z}^{(1)}-\mathbf{Z}^{(2)}\right|}$. Separating the total and relative momenta and coordinates,

$$
\mathbf{R}=\frac{\omega_{1} \mathbf{z}^{(1)}+\omega_{2} \mathbf{z}^{(2)}}{\omega_{1}+\omega_{2}}, \quad \boldsymbol{\eta}=\mathbf{z}^{(1)}-\mathbf{z}^{(2)} ; \quad \boldsymbol{\pi}=\frac{1}{i} \frac{\partial}{\partial \boldsymbol{\eta}}
$$

and $\mathbf{P}=\mathbf{p}^{(1)}+\mathbf{p}^{(2)}$, one obtains in () with $\mathbf{B}=0$

$$
H=\frac{\mathbf{P}^{2}}{2\left(\omega_{1}+\omega_{2}\right)}+\frac{\boldsymbol{\pi}^{2}}{2 \tilde{\omega}}+U(\boldsymbol{\eta})+\sum_{i=1,2} \frac{m_{i}^{2}+\omega_{i}^{2}}{2 \omega_{i}} .
$$

1. The first example is the relativistic electron with mass $m_{1}$ in the Coulomb field of a heavy atom of mass $m_{2}$ with charge $Z e, U(\eta)=-\frac{Z \alpha}{\eta}$. For $\mathbf{P}=0$ one has for the ground state

$$
M\left(\omega_{1}, \omega_{2}\right)=\sum_{i=1,2} \frac{m_{i}^{2}+\omega_{i}^{2}}{2 \omega_{i}}-\frac{\tilde{\omega}(Z \alpha)^{2}}{2}
$$

Minimizing in $\omega_{1}$ for $m_{2} \gg m_{1}$ one obtains

$$
M \approx m_{2}+m_{1} \sqrt{1-(Z \alpha)^{2}}
$$

which coincides with the exact answer from the Dirac equation. 
2. As a second example we consider electron-positron system, then from the same Hamiltonian (56) for $\mathbf{P}=0$ and $m_{1}=m_{2}=m$ one obtains after minimization

$$
M=2 m \sqrt{1-\frac{\alpha^{2}}{4}} \approx 2 m-\frac{m \alpha^{2}}{4}
$$

which looks correct, at least in the expansion in $\alpha$.

3. In the next example we consider the noninteracting $q_{1} \bar{q}_{2}$ system in constant magnetic field $\mathbf{B}$ along the $z$ axis. For $U=0$ one can solve one-body problem for each quark in m.f. with the result for the lowest Landau levels (LLL)

$$
M\left(\omega_{1}, \omega_{2}\right)=\sum_{i} \frac{m_{i}^{2}+\omega_{i}^{2}+e B\left(2 n_{i}+1\right)-e_{i} \boldsymbol{\sigma}^{(i)} \mathbf{B}+\left(\mathbf{p}_{z}^{(i)}\right)^{2}}{2 \omega_{i}}
$$

and after minimization one has

$$
M\left(\omega_{1}^{(0)}, \omega_{2}^{(0)}\right)=\sum_{i} \sqrt{\left(\mathbf{p}_{z}^{(i)}\right)^{2}+m_{i}^{2}+e B\left(2 n_{i}+1\right)-e_{i} \boldsymbol{\sigma}^{(i)} \mathbf{B}}
$$

We turn now to the general case of the $q_{1} \bar{q}_{2}$ system and consider first the case of a neutral system, $e_{1}=-e_{2}=e$. In terms of total and relative momenta the Hamiltonian has the form

$$
\begin{gathered}
H_{q_{1} q_{2}}=H_{B}+H_{\sigma}+U \\
H_{B}=\frac{1}{2 \omega_{1}}\left[\frac{\tilde{\omega}}{\omega_{2}} \mathbf{P}+\boldsymbol{\pi}-\frac{e_{1}}{2} \mathbf{B} \times\left(\mathbf{R}+\frac{\tilde{\omega}}{\omega_{1}} \boldsymbol{\eta}\right)\right]^{2}+ \\
+\frac{1}{2 \omega_{2}}\left[\frac{\tilde{\omega}}{\omega_{1}} \mathbf{P}-\boldsymbol{\pi}-\frac{e_{2}}{2} \mathbf{B} \times\left(\mathbf{R}-\frac{\tilde{\omega}}{\omega_{2}} \boldsymbol{\eta}\right)\right]^{2} \\
H_{\sigma}=\sum_{i=1,2} \frac{m_{i}^{2}+\omega_{i}^{2}-e_{i} \boldsymbol{\sigma}^{(i)} \mathbf{B}}{2 \omega_{i}}
\end{gathered}
$$


The $\mathbf{R}$ dependence for (62) in the case, when $e_{1}=-e_{2}$ can be factorized out in the way, discovered long ago [40]

$$
\Psi(\mathbf{R}, \boldsymbol{\eta})=\varphi(\boldsymbol{\eta}) \exp \left(i \mathbf{P R}-\frac{i e}{2}(\mathbf{B} \times \boldsymbol{\eta}) \mathbf{R}\right)
$$

and for $\varphi(\boldsymbol{\eta})$ one obtains the equation:

$$
\left(H_{0}+H_{\sigma}+U\right) \varphi(\eta)=M \varphi(\eta)
$$

where $H_{0}$ is

$$
H_{0}=\frac{1}{2 \tilde{\omega}}\left(-\frac{\partial^{2}}{\partial \boldsymbol{\eta}^{2}}+\frac{e^{2}}{4}(\mathbf{B} \times \boldsymbol{\eta})^{2}\right) .
$$

In this way $H_{0}$ adds to the confining well with OGE and other terms also an oscillator potential.

One can replace for simplicity the linear confining term by the oscillator potential, $V_{\text {conf }}=\sigma \eta \rightarrow \tilde{V}_{\text {conf }} \equiv \frac{\sigma}{2}\left(\frac{\eta^{2}}{\gamma}+\gamma\right)$, where $\gamma$ satisfies stationary point condition $\left.\frac{\partial M}{\partial \gamma}\right|_{\gamma=\gamma_{0}}=0$, which ensures some $5 \%$ accuracy of this replacement. Then the lowest eigevalue $\bar{M}$ of the basic part of Hamiltonian, $\bar{H}=H_{0}+H_{\sigma}+\tilde{V}_{\text {conf }}$, is

$$
\bar{M}\left(\omega_{1}, \omega_{2}, \gamma\right)=\varepsilon_{n_{\perp}, n_{z}}+\sum_{i=12} \frac{m_{i}^{2}+\omega_{i}^{2}-e_{i} \boldsymbol{\sigma}^{(i)} \mathbf{B}}{2 \omega_{i}},
$$

where $e_{1}=e=-e_{2}$, and

$$
\varepsilon_{n_{\perp}, n_{z}}=\frac{1}{2 \tilde{\omega}}\left[\sqrt{e^{2} B^{2}+\frac{4 \sigma \tilde{\omega}}{\gamma}}\left(2 n_{\perp}+1\right)+\sqrt{\frac{4 \sigma \tilde{\omega}}{\gamma}}\left(n_{z}+\frac{1}{2}\right)\right]+\frac{\gamma \sigma}{2},
$$

We turn now to the case of charged two-body system in m.f., and here one can consider two different situation. In the first case, when $e_{1}=e_{2}=e$ and also $m_{1}=m_{2}$ (and hence $\omega_{1}^{(0)}=\omega_{2}^{(0)}$ ) one can do an exact factorization of $\mathbf{R}$ and $\boldsymbol{\eta}$.

$$
H_{B}=\frac{P^{2}}{2\left(\omega_{1}+\omega_{2}\right)}-\frac{e \mathbf{P}(\mathbf{B} \times \mathbf{R})}{\omega_{1}+\omega_{2}}+\frac{e^{2}}{8 \tilde{\omega}}(\mathbf{B} \times \mathbf{R})^{2}+\frac{\pi^{2}}{2 \tilde{\omega}}+\frac{e^{2}(\mathbf{B} \times \boldsymbol{\eta})^{2}\left(\omega_{1}^{3}+\omega_{2}^{3}\right)}{8\left(\omega_{1}+\omega_{2}\right)^{2} \omega_{1} \omega_{2}}+\Delta H_{B}\left(\omega_{1}, \omega_{2}\right) ;
$$

$H_{\sigma}$ is given in (64), and $\Delta H_{B}$ is 


$$
\begin{gathered}
\Delta H_{B}\left(\omega_{1}, \omega_{2}\right)=-\frac{\omega_{2}^{2}-\omega_{1}^{2}}{\omega_{1} \omega_{2}\left(\omega_{1}+\omega_{2}\right)} \frac{e}{2} \boldsymbol{\pi}(\mathbf{B} \times \boldsymbol{\eta})-\frac{\omega_{2}-\omega_{1}}{\omega_{1} \omega_{2}} \frac{e}{2} \boldsymbol{\pi}(\mathbf{B} \times \mathbf{R})- \\
-\frac{\omega_{2}-\omega_{1}}{\left(\omega_{1} \omega_{2}\right)} \frac{e}{2} \mathbf{P}(\mathbf{B} \times \boldsymbol{\eta})+\frac{\left(\omega_{2}^{2}-\omega_{1}^{2}\right)}{\left(\omega_{1}+\omega_{2}\right)^{2} \omega_{1} \omega_{2}} \frac{e^{2}}{4}(\mathbf{B} \times \mathbf{R})(\mathbf{B} \times \boldsymbol{\eta})
\end{gathered}
$$

For $\omega_{1}=\omega_{2}, \Delta H_{B}$ vanishes and the Hamiltonian has the form

$$
\begin{array}{r}
H=\frac{P^{2}}{4 \omega}-\frac{e(\mathbf{P}(\mathbf{B} \times \mathbf{R}))}{2 \omega}+\frac{e^{2}}{4 \omega}(\mathbf{B} \times \mathbf{R})^{2}+\frac{\pi^{2}}{\omega}+\frac{e^{2}}{16 \omega}(\mathbf{B} \times \boldsymbol{\eta})^{2}+ \\
+\frac{2 m^{2}+2 \omega^{2}-e\left(\boldsymbol{\sigma}_{1}+\boldsymbol{\sigma}_{2}\right) \mathbf{B}}{2 \omega}+\frac{\sigma}{2}\left(\frac{\eta^{2}}{\gamma}+\gamma\right)+V_{\mathrm{OGE}}+V_{s s}+\Delta M_{S E} .
\end{array}
$$

The lowest eigenvalues of the Hamiltonian (172) are

$$
\begin{gathered}
M=\frac{m^{2}+\omega^{2}}{\omega}+\left\langle V_{\mathrm{OGE}}\right\rangle+\left\langle V_{s s}\right\rangle+\left\langle\Delta M_{S E}\right\rangle+ \\
+\frac{e B}{2 \omega}\left(2 N_{\perp}+1\right)+\sqrt{\left(\frac{e B}{2 \omega}\right)^{2}+\frac{2 \sigma}{\gamma_{0} \omega}}\left(2 n_{\perp}+1\right)+\left(n_{\|}+\frac{1}{2}\right) \sqrt{\frac{2 \sigma}{\gamma_{0} \omega}}-\frac{e\left(\boldsymbol{\sigma}_{1}+\boldsymbol{\sigma}_{2}\right) \mathbf{B}}{2 \omega}+\frac{\gamma_{0} \sigma}{2},
\end{gathered}
$$

We now turn to the general case of a charged $q_{1} \bar{q}_{2}$ system, when $e_{1} \neq$ $e_{2}$, and write the full instantaneous Hamiltonian as in (62) - (64), but with arbitrary $e_{1}$ and $e_{2}, e_{1}+e_{2}=e$ and $e$ is the total charge of the meson.

In this case the simple factorization form (65) does not work, and one must instead to make a first step towards factorization, namely one must associate the c.m. motion in m.f. with the total charge $e$ of the system. This is done in the following form, discussed previously in [33]

$$
\begin{gathered}
\Psi(\boldsymbol{\eta}, \mathbf{R})=\exp (i \Gamma) \varphi(\boldsymbol{\eta}, \mathbf{R}), \\
\Gamma=\mathbf{P R}-\frac{\bar{e}}{2}(\mathbf{B} \times \boldsymbol{\eta}) \mathbf{R}, \quad \bar{e}=\frac{e_{1}-e_{2}}{2}
\end{gathered}
$$

and the resulting Hamiltonian from the relation $H_{0} \Psi=\exp (i \Gamma) H_{0}^{\prime} \varphi$, is

$$
H_{0}^{\prime}=\frac{\mathbf{P}^{2}}{2\left(\omega_{1}+\omega_{2}\right)}+\frac{\left(\omega_{1}+\omega_{2}\right) \Omega_{R}^{2} \mathbf{R}_{\perp}^{2}}{2}+\frac{\boldsymbol{\pi}^{2}}{2 \tilde{\omega}}+\frac{\tilde{\omega} \Omega_{\eta}^{2} \boldsymbol{\eta}_{\perp}^{2}}{2}+X_{L P} \mathbf{B} \mathbf{L}_{P}+
$$




$$
\begin{gathered}
+X_{L_{\eta}} \mathbf{B L}_{\eta}+X_{1} \mathbf{P}(\mathbf{B} \times \boldsymbol{\eta})+X_{2}(\mathbf{B} \times \mathbf{R}) \cdot(\mathbf{B} \times \boldsymbol{\eta})+ \\
+X_{3} \boldsymbol{\pi}(\mathbf{B} \times \mathbf{R})+\frac{m_{1}^{2}+\omega_{1}^{2}}{2 \omega_{1}}+\frac{m_{2}^{2}+\omega_{2}^{2}}{2 \omega_{2}} \\
\Omega_{R}^{2}=B^{2} \frac{\left(e_{1}+e_{2}\right)^{2}}{16 \omega_{1} \omega_{2}} \\
\Omega_{\eta}^{2}=\frac{B^{2}}{2 \tilde{\omega}\left(\omega_{1}+\omega_{2}\right)^{2}}\left[\frac{\left(e_{1} \omega_{2}+\bar{e} \omega_{1}\right)^{2}}{2 \omega_{1}}+\frac{\left(e_{2} \omega_{1}-\bar{e} \omega_{2}\right)^{2}}{2 \omega_{2}}\right] .
\end{gathered}
$$

Here all coefficients $X_{i}(i=1,2,3)$ given explicitly in the Appendix 2 of [33].

Treating the terms $X_{1}, X_{2}, X_{3}$ as a perturbation $\Delta M_{X}$,

$$
\Delta M_{X}=\left\langle X_{1} \mathbf{P}(\mathbf{B} \times \boldsymbol{\eta})+X_{2}(\mathbf{B} \times \mathbf{R})(\mathbf{B} \times \boldsymbol{\eta})+X_{3} \boldsymbol{\pi}(\mathbf{B} \times \mathbf{R})\right\rangle,
$$

one can write the total energy eigenvalues $M_{n}^{(0)}$ of the Hamiltonian $\bar{H}$ in (68) as

$$
M_{n}^{(0)}=M^{(0)}(\mathbf{P})+M^{(0)}(\boldsymbol{\pi})+\Delta M_{X}+H_{\sigma}
$$

where

$$
M^{(0)}(\mathbf{P})=\frac{P_{z}^{2}}{2\left(\omega_{1}+\omega_{2}\right)}+\Omega_{R}\left(2 n_{R_{\perp}}+1\right)+X_{L P} \mathbf{L}_{P} \mathbf{B}
$$

$M^{(0)}(\boldsymbol{\pi})$ is the eigenvalue of the operator $H_{\pi}$,

$$
H_{\pi}=\frac{\boldsymbol{\pi}^{2}}{2 \tilde{\omega}}+\frac{\tilde{\omega} \Omega_{\eta}^{2} \boldsymbol{\eta}_{\perp}^{2}}{2}+X_{L_{\eta}} \mathbf{B L}_{\eta}+V_{c o n f}+V_{O G E}
$$

We have written above the most general forms of instantaneous Hamiltonians in the external m.f. It is seen, that to a good accuracy the dynamical contributions of e.m. and color fields can be separated, except in the OGE and spin-dependent terms, and as shown in [38], the m.f. contribution to the both terms is decisive at large $e B$. 


\section{Discussion of results}

We have started with the general $4 \mathrm{~d}$ proper-time path integral for the Green's function of a quark and an antiquark in gluonic $\left(A_{\mu}, F_{\mu \nu}\right)$ and e.m. $\left(A_{\mu}^{e}, B_{\mu \nu}\right)$ fields. These fields are contained in the generalized Wilson loop $W$ with inclusion of spin-field operators $\left(\sigma_{\mu \nu}\left(F_{\mu \nu}+B_{\mu \nu}\right)\right)$.

After vacuum averaging procedure in the partition function, the averaged Wilson loop $\langle W\rangle_{A, A^{(e)}}$ contains all possible interactions, including internal quark loops from the terms $\operatorname{tr} \ln \left(m_{i}^{2}-\hat{D}_{i}^{2}\right)$ in the partition function.

As a first step we have traded the particle proper times for the Euclidean (ordering) times $t_{E}^{(1)}, t_{E}^{(2)}$ and performed path integration over fourth particle coordinates $z_{4}, \bar{z}_{4}$, which is physically the time fluctuations around $t_{E}^{(1)}, t_{E}^{(2)}$. We have shown, that this time-fluctuation integration leads to the $3 \mathrm{~d}$ path integrals with the action (or Hamiltonian in the Hamiltonian form of path integral) which is a matrix in the Fock states. The resulting $3 \mathrm{~d}$ path integrals are integrals over new parameters $\omega_{1}, \omega_{2}$, and the spectrum of the $q_{1} \bar{q}_{2}$ system can be found for large times by a stationary point procedure in $\omega_{1}, \omega_{2}$.

In this way one is going from the $4 \mathrm{~d}$ formalism to the multichannel $3 \mathrm{~d}$ formalism with an additional $\omega$-integration for each particle.

As a next step we have observed that the interaction appearing in the averaged Wilson loop, $\langle W\rangle_{A, A^{(e)}}$, has the form of field correlators $\left\langle F_{\mu \nu}(x) F_{\lambda \sigma}(y)\right\rangle$, $\left\langle B_{\mu \nu}(x) B_{\lambda \sigma}(y)\right\rangle$, and the first correlator has a very small correlation length $\lambda \sim 0.1 \mathrm{fm}$ (found on the lattice [36] and in analytic calculations [37]). This allows to go over to the instantaneous dynamics, when the bolocal or multilocal) interaction $\langle F(x) F(y)\rangle$ is replaced by the time-averaged potentials $V(\mathbf{x}-\mathbf{y})=\int d\left(x_{4}-y_{4}\right)\langle F(x) F(y)\rangle$, and this is valid when the basic parameter, defining the quark trajectory, string tension $\sigma$ satisfies $\sigma \lambda^{2} \ll 1$, so that typical time length on trajectory $t_{0} \sim \frac{1}{\sqrt{\sigma}}$ is much larger than $\lambda$. Note, that this condition is opposite to the one, used for validity of the OPE and QCD sum rules.

As a result one obtains the instantaneous relativistic Hamiltonian $H\left(\omega_{1}, \omega_{2}\right)$ depending on two parameter $\omega_{1}, \omega_{2}$ (for the $\left(q_{1} \bar{q}_{2}\right)$ Hamiltonian matrix element) and the actual spectrum is obtained from the eigenvalues $M_{n}\left(\omega_{1}, \omega_{2}\right)$ at the stationary points $\omega_{1}^{(0)}, \omega_{2}^{(0)}$. Note, that these points are different for different $n=0,1,2 \ldots$

We have checked the results in section 4 for several simple systems and found good agreement with known results. Moreover, this formalism for 
eigenvalues was being used for more than 20 years in many papers, a small part of which was cited here, and the results in all systems, mesons, baryons, hybrids and glueballs are well compared with experimental and lattice ones.

The important new element in this paper is the rigorous derivation of the integral representation for the $\left(q_{1} \bar{q}_{2}\right)$ Green's function Eqs. (41), (42), (48), which gives a new meaning to the parameters $\omega_{1}, \omega_{2}$, and allows not only calculate spectrum, but also the Green's function itself.

As an important application of the developed formalism, we have derived in section 4 the explicit form of Hamiltonians of the $\left(q_{1} \bar{q}_{2}\right)$ system in the constant m.f. B, and defined the main part of the spectrum for neutral and charged mesons.

These results have been used for the explicit numerical evaluation of the $\rho$-meson spectra in [41, which are in reasonable agreement with existing lattice data. Moreover, the same formalism was extensively exploited in [35] for calculation of chiral condensate, and in [33] for magnetic moments.

Actually, the field of possible applications of our method in QCD and QED is enormous, and the method is especially simple in the cases, when only spectral properties are of interest. This is clearly seen, when one compares this method with the Bethe-Salpeter equation. In the last case one is facing the problems of the relative time and insufficiency of the ladder kernel already in the QED case.

In the QCD case the use of the Bethe-Salpeter equation is in addition associated with the vector propagator form of confinement, which is physically not consistent, or with some phenomenological form, and in this way the method loses its fundamental character. On the contrary, the very short-

correlation property of confinement suits perfectly to establish the validity of instantaneous Hamiltonian formalism and allows for an accurate and simple procedure.

The author is grateful to M.A.Andreichikov, A.M.Badalian, S.I.Godunov, B.O.Kerbikov, V.D.Orlovsky, A.E.Shabad, M.I.Vysotsky for many useful discussions.

Appendix 1.

Derivation of the general expression for the $q_{1} \bar{q}_{2}$ Green's function 
We start with the general definition for the $q_{1} \bar{q}_{2}$ Green's function in the vacuum gluonic and external e.m. fields

$$
\begin{gathered}
G(x, y)=\left\langle\operatorname{tr} \Gamma S_{1}(x, y) \bar{\Gamma} \bar{S}_{2}(y, x)\right\rangle_{A}= \\
=\left\langle\operatorname{tr} \Gamma \frac{\left(m_{1}-\hat{D}_{1}\right)}{m_{1}^{2}-\hat{D}_{1}^{2}} \bar{\Gamma} \frac{\left(m_{2}-\hat{\bar{D}}_{2}\right)}{\left(m_{2}^{2}-\hat{\bar{D}}_{2}^{2}\right)}\right\rangle_{A}= \\
=4 \int_{0}^{\infty} d s_{1} \int_{0}^{\infty} d s_{2}\left(D^{4} z^{(1)} D^{4} z^{(2)}\right)_{x y} e^{-K_{1}-K_{2}}\left\langle Y W_{F}\right\rangle,
\end{gathered}
$$

where $\left\langle Y W_{F}\right\rangle=\frac{1}{4} \operatorname{tr}\left[\Gamma\left(m_{1}-i \hat{p}_{1}\right) \bar{\Gamma}\left(m_{2}-i \hat{p}_{2}\right)\left\langle W_{F}\right\rangle_{A}\right]$, and $W_{F} \equiv\langle\langle W\rangle\rangle$, given in (33); the spin operator ordering in (A.12) is not written explicitly. Neglecting spin dependence, one has a purely scalar function $W_{F}$, which is proportional to a unit $(4 \times 4)$ matrix.

Introducing the effective energies $\omega_{i}=\frac{T}{2 s_{i}}, T \equiv\left|x_{4}-y_{4}\right|$, one can rewrite (A.1 1 as

$$
G(x, y)=\frac{T}{2 \pi} \int_{0}^{\infty} \frac{d \omega_{1}}{\omega_{1}^{3 / 2}} \frac{d \omega_{2}}{\omega_{2}^{3 / 2}}\left(D^{3} z^{(1)} D^{3} z^{(2)}\right) \mathbf{x y} e^{-K_{1}\left(\omega_{1}\right)-K_{2}\left(\omega_{2}\right)}\left\langle\left\langle Y W_{F}\right\rangle\right\rangle_{\Delta z_{4}},
$$

and we have taken into account, that

$$
\int\left(D z_{4}^{(1)} D z_{4}^{(2)}\right)_{x_{4} y_{4}}\left\langle Y W_{F}\right\rangle e^{-\frac{1}{4} \int_{0}^{s_{1}}\left(\frac{d z_{4}^{(1)}}{d \tau_{1}}\right)^{2} d \tau_{1}-\frac{1}{4} \int_{0}^{s_{2}}\left(\frac{d z_{4}^{(2)}}{d \tau_{2}}\right)^{2} d \tau_{2}}=\frac{\sqrt{\omega_{1} \omega_{2}}}{2 \pi T}\left\langle\left\langle Y W_{F}\right\rangle\right\rangle_{\Delta z_{4}}
$$

Here $\left\langle\left\langle Y W_{F}\right\rangle\right\rangle_{\Delta z_{4}}$ corresponds to the time-fluctuating Wilson loop average, as in Fig. 1, renormalized and normalized by the condition

$$
\left\langle\left\langle Y W_{F}\right\rangle\right\rangle_{\Delta z_{4}}(g=e=0)=1
$$

We omit in what follows the Fock column structure of the corresponding particle contents in our averaged Wilson loop $\left\langle\left\langle Y W_{F}\right\rangle\right\rangle_{f l}$ with the corresponding $Z_{i}$ factors for each Fock line and concentrate on the simplest case of one renormalized closed $\left(q_{1} \bar{q}_{2}\right)$ loop depending on $t_{E}$, as shown in Fig.2. In the neutral case $e_{1}=-e_{2},\left\langle\left\langle Y W_{F}\right\rangle\right\rangle_{\Delta z_{4}}$ depends only on coordinate differences $\boldsymbol{\eta}\left(t_{E}\right)=\mathbf{z}^{(1)}\left(t_{E}\right)-\mathbf{z}^{(2)}\left(t_{E}\right)$, defined at the same moment $t_{E}$, and one can proceed integrating out the c.m. motion. $K_{1}, K_{2}$ in (A.1 3 ) are

$$
K_{1}\left(\omega_{1}\right)+K_{2}\left(\omega_{2}\right)=\left(\frac{m_{1}^{2}+\omega_{1}^{2}}{2 \omega_{1}}+\frac{m_{2}^{2}+\omega_{2}^{2}}{2 \omega_{2}}\right) T+\int_{0}^{T} d t_{E}\left[\frac{\omega_{1}}{2}\left(\frac{d \mathbf{z}^{(1)}}{d t_{E}}\right)^{2}+\frac{\omega_{2}}{2}\left(\frac{d \mathbf{z}^{(2)}}{d t_{E}}\right)^{2}\right] .
$$


Introducing now the coordinates

$$
\boldsymbol{\eta}\left(t_{E}\right)=\mathbf{z}^{(1)}-\mathbf{z}^{(2)}, \quad \boldsymbol{\rho}\left(t_{E}\right)=\frac{\omega_{1}}{\omega_{1}+\omega_{2}} \mathbf{z}^{(1)}\left(t_{E}\right)+\frac{\omega_{2}}{\omega_{1}+\omega_{2}} \mathbf{z}^{(2)}\left(t_{E}\right)
$$

one can rewrite the last term in (A.1 6 as

$$
\int_{0}^{T} d t_{E}\left(\frac{\omega_{1}+\omega_{2}}{2}\left(\frac{d \boldsymbol{\rho}}{d t_{E}}\right)^{2}+\frac{\tilde{\omega}}{2}\left(\frac{d \boldsymbol{\eta}}{d t_{E}}\right)^{2}\right)
$$

and the path integral $\left(D^{3} z^{(1)} D^{3} z^{(2)}\right) \mathbf{x y}$ as

$$
\begin{aligned}
& \left(D^{3} z^{(1)} D^{3} z^{(2)}\right) \mathbf{x y}=\int \frac{d^{3} \mathbf{p}_{1}}{(2 \pi)^{3}} \int \frac{d^{3} \mathbf{p}_{2}}{(2 \pi)^{3}} e^{i \mathbf{p}_{1}\left(\sum \Delta \mathbf{Z}^{(1)}-(\mathbf{X}-\mathbf{y})\right)+i \mathbf{p}_{2}\left(\sum \Delta \mathbf{Z}^{(2)}-(\mathbf{X}-\mathbf{y})\right)} \times \\
& \times \frac{d^{3} \Delta z^{(1)}}{\left(4 \pi \varepsilon_{1}\right)^{3 / 2}} \frac{d^{3} \Delta z^{(2)}}{\left(4 \pi \varepsilon_{2}\right)^{3 / 2}}=\left(D^{3} \rho\right)_{\mathbf{x y}}\left(D^{3} \eta\right)_{00},
\end{aligned}
$$

Where

$$
\begin{gathered}
\left(D^{3} \boldsymbol{\rho}\right)_{\mathbf{x y}}=\int \frac{d^{3} \mathbf{P}}{(2 \pi)^{3}} \prod_{k} e^{i \mathbf{P}\left(\sum \Delta \boldsymbol{\rho}_{k}-(\mathbf{X}-\mathbf{y})\right)} \frac{d^{3} \Delta \boldsymbol{\rho}_{k}}{\left(\frac{2 \pi \Delta t_{E}}{\omega_{1}+\omega_{2}}\right)^{3 / 2}} \\
\left(D^{3} \boldsymbol{\eta}\right)_{00}=\int \frac{d^{3} \mathbf{q}}{(2 \pi)^{3}} \prod_{k} e^{i \mathbf{q} \sum_{k} \Delta \boldsymbol{\rho}_{k}} \frac{d^{3} \Delta \boldsymbol{\eta}_{k}}{\left(\frac{2 \pi \Delta t_{E}}{\tilde{\omega}}\right)^{3 / 2}} .
\end{gathered}
$$

In absence of external magnetic field, which acts on c.m. coordinate $\boldsymbol{\rho}$, it is convenient to consider the $\mathbf{P}=0$ projection of the Green's function

$$
\begin{gathered}
\int G(x, y) d^{3}(\mathbf{x}-\mathbf{y})=\frac{T}{2 \pi} \int_{0}^{\infty} \frac{d \omega_{1}}{\omega_{1}^{3 / 2}} \int_{0}^{\infty} \frac{d \omega_{2}}{\omega_{2}^{3 / 2}}\left(D^{3} \boldsymbol{\eta}\right)_{00} e^{-K(\eta)}\left\langle\left\langle Y W_{F}\right\rangle\right\rangle_{\Delta z_{4}}= \\
=\frac{T}{2 \pi} \int_{0}^{\infty} \frac{d \omega_{1}}{\omega_{1}^{3 / 2}} \int_{0}^{\infty} \frac{d \omega_{2}}{\omega_{2}^{3 / 2}}\left\langle 0\left|\langle Y\rangle e^{-H T}\right| 0\right\rangle,
\end{gathered}
$$

where

$$
K(\eta)=\left(\frac{m_{1}^{2}+\omega_{1}^{2}}{2 \omega_{1}}+\frac{m_{2}^{2}+\omega_{2}^{2}}{2 \omega_{2}}\right) T+\int_{0}^{T} d t_{E} \frac{\tilde{\omega}}{2}\left(\frac{d \boldsymbol{\eta}}{d t_{E}}\right)^{2}
$$




$$
\left\langle 0\left|e^{-H T}\right| 0\right\rangle=\sum_{n=0}^{\infty}\left|\varphi_{n}(0)\right|^{2} e^{-M_{n}\left(\omega_{1}, \omega_{2}\right) T} .
$$

Here $\varphi_{n}(0)=\left.\varphi_{n}\left(\omega_{1}, \omega_{2}, \boldsymbol{\eta}\right)\right|_{\boldsymbol{\eta}=0}$, and $M_{n}\left(\omega_{1}, \omega_{2}\right)$ is the eigenvalue of the Hamiltonian

$$
H \equiv H\left(\omega_{1}, \omega_{2}\right), \quad H \varphi_{n}=M_{n}\left(\omega_{1}, \omega_{2}\right) \varphi_{n} .
$$

Assuming, that $\left\langle\left\langle W_{F}\right\rangle\right\rangle_{\Delta z_{4}}$ can be represented as

$$
\left\langle\left\langle W_{F}\right\rangle\right\rangle_{\Delta z_{4}}=\exp \left(-\int \hat{V}(\eta, \omega) d t_{E}\right)
$$

the Hamiltonian can be written in the form

$$
H\left(\omega_{1}, \omega_{2}\right)=\sum_{i=1,2} \frac{m_{i}^{2}+\omega_{i}^{2}}{2 \omega_{i}}+\frac{\mathbf{p}^{2}}{2 \tilde{\omega}}+\hat{V}\left(\eta, \omega_{1}, \omega_{2}\right)
$$

At this point one can define the so-called quark decay constants $f_{\Gamma}^{(n)}[30]$,

$$
\left.\int G(x, y) d^{3} \mathbf{x}-\mathbf{y}\right)=\sum_{n} \varepsilon_{\Gamma} \bigotimes \varepsilon_{\Gamma} \frac{\bar{M}_{n}\left(f_{\Gamma}^{(n)}\right)^{2}}{2} e^{-\bar{M}_{n} T}
$$

where $\varepsilon_{\Gamma}=1$ for $S$ and $P$ channels, and $\varepsilon_{\Gamma}=\varepsilon_{\mu}^{(k)}$ for $V, A$ channels,

$$
\sum_{k=1,2,3} \varepsilon_{\mu}^{(k)}(q) \varepsilon_{\nu}^{(k)}(q)=\delta_{\mu \nu}-\frac{q_{\mu} q_{\nu}}{q^{2}}
$$

and hence $f_{\Gamma}^{(n)}$ can be found from (A.1 12) as

$$
\left(f_{\Gamma}^{(n)}\right)^{2} e^{-\bar{M}_{n} T}=\frac{T}{2 \pi} \frac{2\langle Y\rangle}{\bar{M}_{n}} \int_{0}^{\infty} \frac{d \omega_{1}}{\omega_{1}^{3 / 2}} \int_{0}^{\infty} \frac{d \omega_{2}}{\omega_{2}^{3 / 2}} \varphi_{n}^{2}(0) e^{-M_{n}\left(\omega_{1}, \omega_{2}\right) T} .
$$

Here $T$ on both sides is assumed to tend to $\infty$, and one can calculate the integral on the r.h.s. of (A.1 20) by the stationary point method,

$$
\begin{gathered}
M_{n}\left(\omega_{1}, \omega_{2}\right)=M_{n}\left(\omega_{1}^{(0)}, \omega_{2}^{(0)}\right)+M_{n}^{(11)}\left(\omega_{1}^{(0)}, \omega_{2}^{(0)}\right) \frac{\left(\omega_{1}-\omega_{1}^{(0)}\right) 2}{2}+ \\
+M_{n}^{(22)}\left(\omega_{1}^{(0)}, \omega_{2}^{(0)}\right) \frac{\left(\omega_{2}-\omega_{2}^{(0)}\right) 2}{2}+M_{n}^{(12)}\left(\omega_{1}^{(0)}, \omega_{2}^{(0)}\right)\left(\omega_{1}-\omega_{1}^{(0)}\right)\left(\omega_{2}-\omega_{2}^{(0)}\right)
\end{gathered}
$$


where

$$
M_{n}^{(i k)}=\left.\frac{\partial^{2} M_{n}}{\partial \omega_{i} \partial \omega_{k}}\right|_{\omega_{i}=\omega_{i}^{(0)}, \omega_{k}=\omega_{k}^{(0)}},
$$

and

$$
\left.\frac{\partial M_{n}}{\partial \omega_{i}}\right|_{\omega_{i}=\omega_{i}^{(0)}}=0, i=1,2 .
$$

Doing the integration in (A.1 20) with the help of (A.1 21) one obtains

$$
\left(f_{\Gamma}^{(n)}\right)^{2}=\frac{N_{c}\langle Y\rangle \varphi_{n}^{2}(0)}{\left(\omega_{1}^{(0)} \omega_{2}^{(0)}\right) \bar{M}_{n} \xi_{n}}
$$

where

$$
\xi_{n}=\sqrt{\omega_{1}^{(0)} \omega_{2}^{(0)} \Omega_{n}}
$$

with

$$
\Omega_{n}=\frac{\alpha \beta(\alpha-\beta)^{2}}{(\alpha-\beta)^{2}+\gamma^{2}}+\frac{\gamma^{2}\left[(\alpha+\beta)^{2}-2(\alpha-\beta)^{2}-\gamma^{2}\right]}{4\left[(\alpha-\beta)^{2}+\gamma^{2}\right]}
$$

where we have denoted

$$
\alpha=\frac{1}{2} M_{n}^{(11)}, \quad \beta=\frac{1}{2} M_{n}^{(22)}, \quad \gamma=M_{n}^{(12)},
$$

and finally

$$
\bar{M}_{n}=M_{n}\left(\omega_{1}^{(0)}, \omega_{2}^{(0)}\right), \quad \bar{Y}=\frac{1}{4} \operatorname{tr}_{D}\left(\Gamma\left(m_{1}-i \hat{p}_{1}\right) \bar{\Gamma}\left(m_{2}-i \hat{p}_{2}\right)\right.
$$

and $\operatorname{tr}_{D}$ denotes trace over Dirac $4 \times 4$ indices. It is instructive to compare (A.1 24) with the old result, obtained in [30], using approximate path integrals over $(D \Delta \omega)$,

$$
\left(f_{\Gamma}^{(n)}\right)_{\Delta \omega}^{2}=\frac{2 N_{c}\langle Y\rangle \varphi_{n}^{2}(0)}{\bar{M}_{n} \omega_{1}^{(0)} \omega_{2}^{(0)}} .
$$

As one can see, comparing A.1 24 and A.1 27), in the first case (the time-fluctuation approach of the present paper) the factor $\frac{1}{\sqrt{\Omega \omega_{1}^{(0)} \omega_{2}^{(0)}}}$ should be equal to 2 , for both expressions to coincide. In practice for the $\left(q_{1} \bar{q}_{2}\right)$ state 
made of zero mass quarks, $m_{1}=m_{2}=0$, and with the total mass made of confining interaction, see [28] for details, one has

$$
M_{n}\left(\omega_{1}, \omega_{2}\right)=\sum_{i=1}^{2} \frac{m_{i}^{2}+\omega_{i}^{2}}{2 \omega_{i}}+(2 \tilde{\omega})^{-1 / 3} \sigma^{2 / 3} a_{n}, a_{0}=2,338
$$

and for $m_{1}=m_{2}=0$ one obtains

$$
\left(\Omega_{0} \omega_{1}^{(0)} \omega_{2}^{(0)}\right)^{-1 / 2}=3,
$$

while for $m_{1}=0, m_{2} \ll \sqrt{\sigma}$, the result is

$$
\left(\Omega_{0} \omega_{1}^{(0)} \omega_{2}^{(0)}\right)^{-1 / 2} \cong 2.34 .
$$

This implies, that the quark decay constants $f_{\Gamma}^{(n)}$ obtained in the new method will be larger by (10-20)\% as compared with previous calculations in [30].

\section{References}

[1] R.P.Feynman, Rev. Mod. Phys. 20, 367 (1948).

[2] R.P.Feynman and A.R.Hibbs, Quantum Mechanics and Path Integrals, (McGraw-Hill, New York, 1965).

[3] A.M.Polyakov, Gauge Fields and Strings (Harwood Academic, 1987);

H.M.Fried, Functional Methods and Models in Quantum Field Theory (MIT Press, Cambridge, 1972);

L.S.Schulman, Techniques and Applications of Path integration, (John Wiley, New York, 1981).

[4] H.Kleinert, Path Integrals in Quantum Mechanics, Statistics and Polymer Physics, 2nd ed., (World Scientific, Singapore, 1995);

M.Reuter, M.G.Schmidt and C.Schubert, Ann. Phys. (NY), 259, 313 (1997);

C.Grosche and F.Steiner, Handbook of Feynman Path Integrals (Springer-Verlag, Berlin-Heidelberg, 1998). 
[5] N.N.Bogolyubov and D.V.Shirkov, Introduction to the Theory of Quantum Fields, (Nauka, Moscow, 1976);

A.A.Slavnov, Teor. Mat. Fiz. 22, 177 (1975);

L.D.Faddeev and A.A.Slavnov, Introduction to the Theory of Quantized Fields, (Nauka, Moscow, 1978).

[6] C.Itzykson and J.-B.Zuber, Quantum Field Theory, (McGraw-Hill, New York, 1980).

[7] T.D.Lee, Particle Physicks and Introduction to Field Theory, (Harwood Academic, 1981).

[8] M.E.Peskin and D.V.Schroeder, An Introduction to Quantum Field Theory, Addison-Wesley, 1995.

[9] F.J.Yndurain, The Theory of Quark and Gluon Interactions, (4th ed., Springer, 2006).

[10] V.A.Fock, Izv. Akad. Nauk USSR, OMEN, 557 (1937).

[11] J.S.Schwinger, Phys. Rev. 82, 664 (1951).

[12] R.P.Feynman, Phys. Rev. 80, 440 (1950); ibid 84, 108 (1951).

[13] G.A.Mikhlin and E.S.Fradkin, ZhETF 45, 1926 (1963); E.S.Fradkin, Trudy FIAN 29, 7 (1965);

E.S.Fradkin, Nucl. Phys. 76, 588 (1966);

F.A.Berezin and M.S.Marinov, Ann. Phys. (NY),104, 336 (1977).

[14] M.B.Halpern and P.Senjanivic, Phys. Rev. D 15, 1655 (1977);

M.B.Halpern, A.Jevicki and P.Senjanivic, Phys. Rev. D 16, 2474 (1977).

[15] R.Brandt et al. Phys. Rev. D 19, 1153 (1979).

[16] S.Samuel, Nucl. Phys. B149, 517 (1979);

J.Ishida and A.Hosoya, Progr. Theor. Phys. 62, 544 (1979);

F.Bordi and R.Casalbuoni, Phys. Lett. B93, 308 (1980);

A.I.Karanicas and C.N.Ktorides, Phys. Lett. B 275, 403 (1992);

A.I.Karanicas, C.N.Ktorides, and N.G.Stefanis, Phys. Rev. D 52, 5898 (1995).

[17] Yu.A.Simonov, Nucl. Phys. B 307, 512 (1988). 
[18] Yu.A.Simonov, Yad. Fiz. 54, 192 (1991).

[19] Yu. A.Simonov, Phys. At. Nucl. 58, 309 (1995):hep-ph/9311216.

[20] Yu.A.Simonov and J.A.Tjon, Ann. Phys. (N.Y.) 228, 1 (1993).

[21] Yu.A.Simonov and J.A.Tjon, Ann. Phys. (N.Y.) 300, 54 (2002).

[22] Yu.A.Simonov, Nucl. Phys. B324, 67 (1989).

[23] A.Yu.Dubin, A.B.Kaidalov and Yu.A.Simonov, Phys. Lett. B343,310 (1995); Phys. At. Nucl. 58, 300 (1995);

Yu.S.Kalashnikova, A.V.Nefediev and Yu.A.Simonov, Phys. Rev. D64, 014037 (2001).

[24] Yu.A.Simonov, Phys. Lett. B249, 514 (1990);

[25] A.B.Kaidalov and Yu.A.Simonov, Phys. At. Nucl. 63,1428 (200); Phys. Lett. B 636, 101 (2006); Yu.A.Simonov, Phys. At. Nucl. 70, 44 (2007).

[26] L.Brink, P.Di Vecchia and P.Howe, Nucl. Phys. B118, 76 (1977);

Yu.A.Simonov, Phys. Lett. B226, 151 (1989); Yu.S.Kalashnikova and A.V.Nefediev, Phys. At. Nucl. 60, 1389 (1997).

[27] A.Giacomo, H.G.Dosch, V.I.Shevchenko and Yu.A.Simonov, Phys. Rept. 372, 319 (2002).

[28] Yu.A.Simonov, QCD and Theory of Hadrons in: "QCD: Perturbative or Nonperturbative." Interscience, Singapore, 2000; hep-ph/9911237.

[29] A.M.Badalian, Yu.A.Simonov and V.I.Shevchenko, Phys.At. Nucl. 69, $1781(2006)$.

[30] A.M.Badalian, B.L.G.Bakker, Yu.A.Simonov Phys. Rev. D75, 116001 (2007).

[31] Yu.A.Simonov, Phys. Lett. B719, 464 (2013).

[32] M.A.Andreichikov, B.O.Kerbikov and Yu.A.Simonov, arXiv:1210.0227, [hep-ph].

[33] A.M.Badalian and Yu.A.Simonov, arXiv:1211.4349, [hep-ph]. 
[34] M.A.Andreichikov, V.D.Orlovsky and Yu.A.Simonov, arXiv:1211.6568, [hep-ph].

[35] Yu.A.Simonov, arXiv:1212.3118, [hep-ph].

[36] A. Di Giacomo and H.Panagopoulos, Phys. Lett. B 285, 133 (1992); A. Di Giacomo, E Meggiolaro and H.Panagopoulos, Nucl. Phys. B483, 371 (1997);

I.Jorysz and C.Michael, Nucl. Phys., 302, 448 (1988).

[37] Yu.A.Simonov, Nucl. Phys. B592, 350 (2001);

Yu.A.Simonov, arXiv:1003.3608;

Yu.A.Simonov and V.I.Shevchenko, Adv. HEPh, 873051 (2009), arXiv:0902.1405.

[38] Yu.A.Simonov, Spin interactions in mesons in strong magnetic field (in preparation).

[39] Yu.A.Simonov, Phys. Lett., B515, 137 (2001);

A. Di Giacomo and Yu.A.Simonov, Phys. Lett., B595, 368 (2004).

[40] W.E.Lamb, Phys. Rev. 85, 259 (1952);

L.P.Gor'kov and I.E.Dzyaloshinskii, Sov. Phys. JETP, 26, 449 (1968);

H.Grotsch and R.A.Hegstrom, Phys. Rev. A 4, 59 (1971);

J.E.Arron, I.E.Herbst, B.Simon, Ann. Phys. (NY) 114, 431 (1978).

[41] M.A.Andreichikov, B.O.Kerbikov, V.D.Orlovsky and Yu.A.Simonov, (in preparation). 